"This is a post-peer-review, pre-copyedit version of an article published in Kacprzyk, J.; Szmidt, E.; Zadrożny, S.; Atanassov, K.; Krawczak, M. (eds) Advances in Fuzzy Logic and Technology 2017. Advances in Intelligent Systems and Computing, vol. 642. Cham: Springer, 2017, p. 143-153. The final authenticated version is available online at: http://dx.doi.org/10.1007/978-3-319-66824-6_13".

\title{
Clustering U.S. 2016 presidential candidates through linguistic appraisals
}

\author{
Raquel González del Pozo ${ }^{1}$, José Luis García-Laprestaaํㄹ David Pérez-Román ${ }^{3}$ \\ ${ }^{1}$ PRESAD Research Group, IMUVA, Departamento de Economía Aplicada, \\ Universidad de Valladolid, Spain \\ raquel.gonzalez.pozo@uva.es \\ ${ }^{2}$ PRESAD Research Group, BORDA Research Unit, IMUVA, Departamento de \\ Economía Aplicada, Universidad de Valladolid, Spain \\ laprestad@eco.uva.es \\ ${ }^{3}$ PRESAD Research Group, BORDA Research Unit, Departamento de Organización \\ de Empresas y Comercialización e Investigación de Mercados, Universidad de \\ Valladolid, Spain \\ david@emp.uva.es
}

\begin{abstract}
During electorate campaigns, linguistic appraisals of presidental candidates given by voters are essential. In order to deal with linguistic appraisals and cluster analysis, this paper presents the results of grouping the United States 2016 presidential candidates using linguistic appraisals collected from a political survey. To do this, we have developed an agglomerative hierarchical clustering procedure based on consensus through the concept of ordinal proximity measure.
\end{abstract}

Keywords: Clustering, Presidential candidates, Ordinal proximity measures, Consensus, Linguistic appraisals

\section{Introduction}

One of the main issues in many research fields is to classify a collection of data into meaningful groups. Cluster analysis is a multivariate method which tries to classify a sample of observations into similar groups regarding given characteristics. This technique is used in several disciplines: Economics, Marketing, Biology, Political Science, Artificial Intelligence, Computer Science, Engineering, etc.

In the political context, clustering has hardly considered the views of the electorate. This method has been applied mainly to identify relationships among demographically similar voters, voting tendencies, as well as patterns of political parties support in different elections (see Seabrook [13], Pearson and Cooper [11] and Aleskerov and Nurmi [1], among others).

Appraisals of candidates given by voters are essential during presidential races, being easy to find linguistic scales in political surveys for evaluating candidates. In recent years, there have arisen voting systems based on linguistic 
appraisals. For instance, Balinski and Laraki [2,3] introduce the Majority Judgment voting system, where voters assign a linguistic term of the following ordered qualitative scale: 'reject', 'poor', 'acceptable', 'good', 'very good' and 'excellent' to each candidate. However, there is so far little literature addressing cluster analysis applied to linguistic appraisals.

The main purpose of this paper is to cluster the United States (U.S.) 2016 presidential candidates taking the linguistic appraisals made by a random representative sample of adults living in the U.S. as our starting point. To do this, we have used the concept of ordinal proximity measure (see García-Lapresta and Pérez-Román [8]), which allows to determine the degree of consensus in a group of agents when a set of alternatives is evaluated through non-necessarily qualitative scales.

The rest of the paper is organized as follows. Section 2 contains notation and some applications of ordinal proximity measures to consensus and clustering. Section 3 includes the results of clustering U.S. 2016 presidential candidates through linguistic appraisals. Finally, Section 4 concludes with some remarks.

\section{Consensus and clustering}

In this section we introduce the concept of ordinal proximity measure and we tackle some applications of this concept to consensus and clustering.

\subsection{Preliminaries}

Consider a set of agents $A=\{1, \ldots, m\}$, with $m \geq 2$, and a set of alternatives $X=\left\{x_{1}, \ldots, x_{n}\right\}$, with $n \geq 2$, which have to be appraised. Each agent assigns a linguistic term to every alternative within an ordered qualitative scale $\mathcal{L}=$ $\left\{l_{1}, \ldots, l_{g}\right\}$, arranged from the lowest to the highest terms, where the granularity of $\mathcal{L}$ is at least $3(g \geq 3)$.

We now recall the notion of ordinal proximity measure, introduced by GarcíaLapresta and Pérez-Román [8], which is a mapping that assigns an ordinal degree of proximity to each pair of linguistic terms of an ordered qualitative scale. These ordinal degrees of proximity belong to a linear order $\Delta=\left\{\delta_{1}, \ldots, \delta_{h}\right\}$ with $\delta_{1} \succ \cdots \succ \delta_{h}$, being $\delta_{1}$ and $\delta_{h}$ the maximum and the minimum degrees of proximity, respectively. The elements of $\Delta$ have no meaning, they only represent different degrees of proximity.

Definition 1. ([8]) $A n$ ordinal proximity measure on $\mathcal{L}$ with values in $\Delta$ is a mapping $\pi: \mathcal{L}^{2} \longrightarrow \Delta$, where $\pi\left(l_{r}, l_{s}\right)=\pi_{r s}$ means the degree of proximity between $l_{r}$ and $l_{s}$, satisfying the following conditions:

1. Exhaustiveness: For every $\delta \in \Delta$, there exist $l_{r}, l_{s} \in \mathcal{L}$ such that $\delta=\pi_{r s}$.

2. Symmetry: $\pi_{s r}=\pi_{r s}$, for all $r, s \in\{1, \ldots, g\}$.

3. Maximum proximity: $\pi_{r s}=\delta_{1} \Leftrightarrow r=s$, for all $r, s \in\{1, \ldots, g\}$.

4. Monotonicity: $\pi_{r s} \succ \pi_{r t}$ and $\pi_{s t} \succ \pi_{r t}$, for all $r, s, t \in\{1, \ldots, g\}$ such that $r<s<t$. 
Every ordinal proximity measure can be represented by a $g \times g$ symmetric matrix with coefficients in $\Delta$, where the elements in the main diagonal are $\pi_{r r}=$ $\delta_{1}, r=1, \ldots, g$ :

$$
\left(\begin{array}{ccccc}
\pi_{11} & \cdots & \pi_{1 s} & \cdots & \pi_{1 g} \\
\cdots & \cdots & \cdots & \cdots & \cdots \\
\pi_{r 1} & \cdots & \pi_{r s} & \cdots & \pi_{r g} \\
\cdots & \cdots & \cdots & \cdots & \cdots \\
\pi_{g 1} & \cdots & \pi_{g s} & \cdots & \pi_{g g}
\end{array}\right)
$$

This matrix is called proximity matrix associated with $\pi$.

\subsection{Consensus}

To determine the degree of consensus we follow the approach introduced by García-Lapresta and Pérez-Román [8]. We start from the appraisals of alternatives given by agents, collected in a profile, that is a matrix

$$
V=\left(\begin{array}{ccccc}
v_{1}^{1} & \cdots & v_{i}^{1} & \cdots & v_{n}^{1} \\
\cdots & \cdots & \cdots & \cdots & \cdots \\
v_{1}^{a} & \cdots & v_{i}^{a} & \cdots & v_{n}^{a} \\
\cdots & \cdots & \cdots & \cdots & \cdots \\
v_{1}^{m} & \cdots & v_{i}^{m} & \cdots & v_{n}^{m}
\end{array}\right)=\left(v_{i}^{a}\right)
$$

consisting of $m$ rows and $n$ columns of linguistic terms, where the element $v_{i}^{a} \in \mathcal{L}$ represents the linguistic appraisal given by the agent $a \in A$ to the alternative $x_{i} \in X$.

According to García-Lapresta and Pérez-Román [10], for measuring the consensus in a group of agents over a set of alternatives, the first step is to consider the degrees of ordinal proximity between the linguistic appraissals over the alternatives. These degrees are arranged in a vector of ordinal degrees $\boldsymbol{\delta} \in \Delta^{p}$, for some $p \in \mathbb{N}$, from the highest to the lowest degrees (decreasing fashion).

In order to avoid loss of information, García-Lapresta and Pérez-Román [10] select the median(s) of the mentioned ordinal degrees in such a way that if the number of ordinal degrees of the vector is odd, the median is unique, $\delta_{r} \in \Delta$, but if the number of ordinal degrees is even, then $\boldsymbol{\delta}$ has two medians, $\delta_{s}, \delta_{t} \in \Delta$ with $s \leq t$. In order to unify this assignment of medians, the authors consider the pair of medians $\left(\delta_{r}, \delta_{r}\right)$ in the odd case and $\left(\delta_{s}, \delta_{t}\right)$ in the even case.

Given the set of feasibles medians $\Delta_{2}=\left\{\left(\delta_{r}, \delta_{s}\right) \in \Delta^{2} \mid r \leq s\right\}$, the median operator is the mapping

$$
M: \bigcup_{p=1}^{\infty} \Delta^{p} \longrightarrow \Delta_{2}
$$

that assigns the corresponding pair of medians to each vector of ordinal degrees.

We denote with \#I the cardinality of $I$ and with $\mathcal{P}_{2}(A)=\{I \subseteq A \mid \# I \geq 2\}$ the family of subsets of at least two agents. 
Definition 2. Given a profile $V=\left(v_{i}^{a}\right)$, the degree of consensus in a subset of agents $I \in \mathcal{P}_{2}(A)$ over a subset of alternatives $\emptyset \neq Y \subseteq X$ is defined as

$$
C(I, Y)=M\left(\pi\left(v_{i}^{a}, v_{i}^{b}\right)_{\substack{a, b \in I, a<b \\ x_{i} \in Y}}\right) \in \Delta_{2} .
$$

For comparing the degrees of consensus in a group of agents $I$ when they evaluate two subsets of alternatives $Y$, $Z$, i.e. $C(I, Y) \in \Delta_{2}$ versus $(I, Z) \in \Delta_{2}$, an appropriate linear order on $\Delta_{2}$ is required. In this paper we use the following linear order used by García-Lapresta and Pérez-Román [10]:

$$
\left(\delta_{r}, \delta_{s}\right) \succeq\left(\delta_{t}, \delta_{u}\right) \Leftrightarrow\left\{\begin{array}{l}
r+s<t+u \\
\text { or } \\
r+s=t+u \text { and } s-r \leq u-t
\end{array}\right.
$$

for all $\left(\delta_{r}, \delta_{s}\right),\left(\delta_{t}, \delta_{u}\right) \in \Delta_{2}$.

It is important to note that the above linear order for ranking the medians is not the only one that can be considered on $\Delta_{2}$.

Since the cardinality of $\Delta_{2}$ may be low, it is very easy to have ties among the degrees of consensus in different subsets of agents or alternatives. In these cases, we resort to a sequential tie-breaking procedure [2] which consists of removing the median(s) of the respective agents or alternatives that are in a tie, and select again the new median(s) of remaining ordinal degrees until ties are broken.

Starting from $C^{(1)}(I, Y)=C(I, Y)$, we calculate $C^{(2)}(I, Y)$ as in $C(I, Y)$ but after dropping the pair of medians of the list $\pi\left(v_{i}^{a}, v_{i}^{b}\right)_{a, b \in I, a<b}$, and analogously for $C^{(3)}(I, Y)$, etc.

$$
x_{i} \in Y
$$

\subsection{Clustering}

The goal of clustering methodology is to reduce the number of objects by classifying them into a set of groups with similar features.

There are many types of clustering and applications (see Everitt et al. [5]) being the agglomerative hierarchical clustering the method used in this paper. This process starts by assigning each item to its own cluster and then, clusters are sequentially merged according to a given similarity criterion, until all of them end up in the same cluster.

The first steps in cluster analysis are to determine the variables and the criterion that will be used for joining clusters or elements. In general, many clustering procedures are based on distances to determine the similarity in a set of elements. However, in this paper we have taken into account the degree of consensus as similarity measure in order to merge clusters. For this purpose, we have considered a similarity function and a sequential similarity vector based on the degree of consensus $[7,9]$, in such a way that the consensus is measured by means of the degrees of proximity between all pairs of individual appraisals over the evaluated alternatives. 
Definition 3. Given a profile $V=\left(v_{i}^{a}\right)$, the similarity function relative to a subset of agents $I \in(\mathcal{P}(A) \backslash\{\emptyset\})$

$$
S_{I}:(\mathcal{P}(X) \backslash\{\emptyset\})^{2} \longrightarrow \Delta_{2}
$$

is defined as

$$
S_{I}(Y, Z)= \begin{cases}C(I, Y \cup Z), & \text { if } \#(Y \cup Z) \geq 2, \\ \left(\delta_{1}, \delta_{1}\right), & \text { if } \#(Y \cup Z)=1 .\end{cases}
$$

Definition 4. Given a profile $V=\left(v_{i}^{a}\right)$, the sequential similarity vector relative to a subset of agents $I \in(\mathcal{P}(A) \backslash\{\emptyset\})$ for $\emptyset \neq Y, Z \subseteq X$ is defined as

$$
\boldsymbol{S}_{I}(Y, Z)=\left(S_{I}^{(1)}(Y, Z), S_{I}^{(2)}(Y, Z), \ldots\right)
$$

where

$$
S_{I}^{(k)}(Y, Z)= \begin{cases}C^{(k)}(I, Y \cup Z), & \text { if } \#(Y \cup Z) \geq 2, \\ \left(\delta_{1}, \delta_{1}\right), & \text { if } \#(Y \cup Z)=1\end{cases}
$$

The agglomerative hierarchical clustering procedure is related to the one provided by García-Lapresta and Pérez-Román [8]. It consists of a sequential process addressed by the following stages:

1. Given a subset of agents $I \in(\mathcal{P}(A) \backslash\{\emptyset\})$, the initial clustering is $\mathcal{A}_{0}^{I}=$ $\left\{\left\{x_{1}\right\}, \ldots,\left\{x_{n}\right\}\right\}$.

2. Calculate the similarities between all the pairs of alternatives $S_{I}\left(\left\{x_{i}\right\},\left\{x_{j}\right\}\right)$ for all $x_{i}, x_{j} \in X$.

3. Select the two alternatives $x_{i}, x_{j} \in X$ that maximize $\boldsymbol{S}_{I}$ (taking into account the corresponding sequential similarity vectors) and construct the first cluster $\mathcal{A}_{1}^{I}=\left\{x_{i}, x_{j}\right\}$.

4. The new clustering is $\mathcal{A}_{1}^{I}=\left(\mathcal{A}_{0}^{I} \backslash\left\{\left\{x_{i}\right\},\left\{x_{j}\right\}\right\}\right) \cup\left\{A_{1}^{I}\right\}$.

5. Calculate the similarities $S_{I}\left(A_{1}^{I},\left\{x_{k}\right\}\right)$ and take into account the previously computed similarities $S_{I}\left(\left\{x_{k}\right\},\left\{x_{l}\right\}\right)$, for all $\left\{x_{k}\right\},\left\{x_{l}\right\} \in \mathcal{A}_{1}^{I}$

6. Select the two alternatives of $\mathcal{A}^{I}$ that maximize $\boldsymbol{S}_{I}$ and to construct the second cluster $A_{2}^{I}$

7. Proceed as in previous items until obtaining the next clustering $\mathcal{A}_{2}^{I}$.

The process continues in the same way until obtaining the last cluster, $\mathcal{A}_{n-1}^{I}=\{X\}$. 


\section{Clustering presidential candidates}

Recently, the issue of clustering presidential candidates of U.S. has been tackled in some publications. For instance, Dowdle et al. [4] gather Republican and Democratic candidates of the parties according to the multiple donor networks, regardless of voters' appraisals.

In this section, taking linguistic appraisals as starting point, we have developed an agglomerative hierarchical clustering in the context of the U.S. 2016 presidential elections. For this purpose, we have considered some data from the January 2016 Political Survey conducted by the Pew Research Center [12], which provides information about political values, domestic policy issues and the interests of U.S. electorate.

In particular, we have focused on a question in which a total of 1184 individuals appraised the nine U.S. presidential candidates included in Table 1, in case of being elected in November 2016. To do this, the individuals used the linguistic terms of the qualitative scale contained in Table 2.

As has been previously explained in Subsection 2.3, the clustering procedure is based on the similarity between alternatives with respect to a group of agents. Therefore, the candidates (alternatives) are classified into different clusters when they maximize the degree of consensus among the agents.

\begin{tabular}{cl} 
Agents Name \\
\hline 1 & Ben Carson \\
2 & Bernie Sanders \\
3 & Chris Christie \\
4 & Donald Trump \\
5 & Hillary Clinton \\
6 & Jeb Bush \\
7 & John Kasich \\
8 & Marco Rubio \\
9 & Ted Cruz \\
\hline Table & 1. Candidates.
\end{tabular}

$\overline{l_{1}}$ Terrible president

$l_{2}$ Poor president

$l_{3}$ Average president

$l_{4}$ Good president

$l_{5}$ Great president

Table 2. Linguistic terms in $\mathcal{L}$.

In order to show the clustering process when the proximities between the linguistic terms of the scale are different, we considered the three most reasonable 
cases in accordance with the qualitative scale used in the survey question. First, the uniform case, where the proximities between consecutive linguistic terms are always the same, and two non-uniform cases in which $l_{2}$ and $l_{4}$ are considered symmetric with respect to $l_{3}$.

The ordinal proximity measures and the clusters of candidates related to each cases are shown below.

1. The uniform case is represented in the following upper half proximity matrix, where the subindices of the matrix correspond to the subindices of the $\delta$ 's appearing just over the main diagonal and denoting the proximity between consecutive terms of the scale.

$$
A_{2222}=\left(\begin{array}{rrrrr}
\delta_{1} & \delta_{2} & \delta_{3} & \delta_{4} & \delta_{5} \\
& \delta_{1} & \delta_{2} & \delta_{3} & \delta_{4} \\
& \delta_{1} & \delta_{2} & \delta_{3} \\
& \delta_{1} & \delta_{2} \\
& & \delta_{1}
\end{array}\right)
$$

This case can be visualized in Figure 1 .

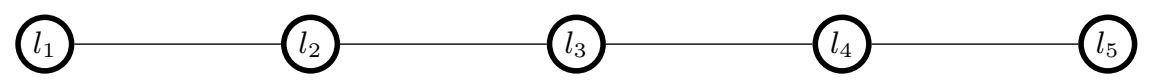

Fig. 1. Ordinal proximity measure with associated matrix $A_{2222}$.

After comparing the different sequential similarity vectors ${ }^{1}$, we have obtained the following clusters of candidates:

$$
\begin{aligned}
& \mathcal{A}_{0}^{A}=\{\{1\},\{2\},\{3\},\{4\},\{5\},\{6\},\{7\},\{8\},\{9\}\} \\
& \mathcal{A}_{1}^{A}=\{\{1\},\{2\},\{3,7\},\{4\},\{5\},\{6\},\{8\},\{9\}\} \\
& \mathcal{A}_{2}^{A}=\{\{1\},\{2\},\{3,6,7\},\{4\},\{5\},\{8\},\{9\}\} \\
& \mathcal{A}_{3}^{A}=\{\{1\},\{2\},\{3,6,7,8\},\{4\},\{5\},\{9\}\} \\
& \mathcal{A}_{4}^{A}=\{\{1,3,6,7,8\},\{2\},\{4\},\{5\},\{9\}\} \\
& \mathcal{A}_{5}^{A}=\{\{1,3,6,7,8,9\},\{2\},\{4\},\{5\}\} \\
& \mathcal{A}_{6}^{A}=\{\{1,2,3,6,7,8,9\},\{4\},\{5\}\} \\
& \mathcal{A}_{7}^{A}=\{\{1,2,3,5,6,7,8,9\},\{4\}\} \\
& \mathcal{A}_{8}^{A}=\{\{1,2,3,4,5,6,7,8,9\}\} .
\end{aligned}
$$

\footnotetext{
${ }^{1}$ The computations for obtaining the corresponding sequential consensus and similarity vectors have been performed with MATLAB.
} 
2. The non-uniform case in which $l_{2}$ and $l_{4}$ are considered further away from $l_{3}$ is associated with the matrix

$$
A_{2332}=\left(\begin{array}{rrrrr}
\delta_{1} & \delta_{2} & \delta_{4} & \delta_{6} & \delta_{7} \\
\delta_{1} & \delta_{3} & \delta_{5} & \delta_{6} \\
& \delta_{1} & \delta_{3} & \delta_{4} \\
& \delta_{1} & \delta_{2} \\
& & \delta_{1}
\end{array}\right)
$$

that can be visualized in Figure 2.

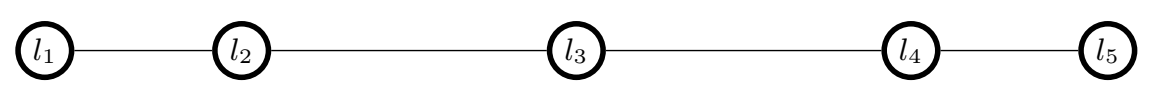

Fig. 2. Ordinal proximity measure with associated matrix $A_{2332}$.

$$
\begin{aligned}
& \mathcal{A}_{0}^{A}=\{\{1\},\{2\},\{3\},\{4\},\{5\},\{6\},\{7\},\{8\},\{9\}\} \\
& \mathcal{A}_{1}^{A}=\{\{1\},\{2\},\{3\},\{4,7\},\{5\},\{6\},\{8\},\{9\}\} \\
& \mathcal{A}_{2}^{A}=\{\{1\},\{2\},\{3,6\},\{4,7\},\{5\},\{8\},\{9\}\} \\
& \mathcal{A}_{3}^{A}=\{\{1,4,7\},\{2\},\{3,6\},\{5\},\{8\},\{9\}\} \\
& \mathcal{A}_{4}^{A}=\{\{1,4,7\},\{2\},\{3,6,8\},\{5\},\{9\}\} \\
& \mathcal{A}_{5}^{A}=\{\{1,3,4,6,7,8\},\{2\},\{5\},\{9\}\} \\
& \mathcal{A}_{6}^{A}=\{\{1,3,4,6,7,8,9\},\{2\},\{5\}\} \\
& \mathcal{A}_{7}^{A}=\{\{1,2,3,4,6,7,8,9\},\{5\}\} \\
& \mathcal{A}_{8}^{A}=\{\{1,2,3,4,5,6,7,8,9\}\} .
\end{aligned}
$$

3. The non-uniform case in which $l_{2}$ and $l_{4}$ are considered closer to $l_{3}$ is associated with the matrix

$$
A_{3223}=\left(\begin{array}{rrrrr}
\delta_{1} & \delta_{3} & \delta_{5} & \delta_{6} & \delta_{7} \\
& \delta_{1} & \delta_{2} & \delta_{4} & \delta_{6} \\
& \delta_{1} & \delta_{2} & \delta_{5} \\
& \delta_{1} & \delta_{3} \\
& & \delta_{1}
\end{array}\right)
$$

that can be visualized in Figure 3 . 


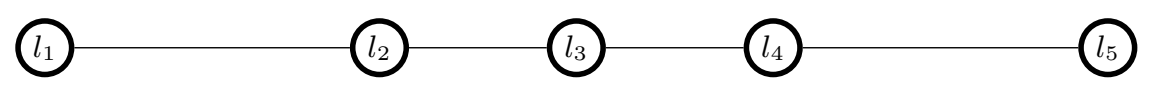

Fig. 3. Ordinal proximity measure with associated matrix $A_{3223}$

$$
\begin{aligned}
& \mathcal{A}_{0}^{A}=\{\{1\},\{2\},\{3\},\{4\},\{5\},\{6\},\{7\},\{8\},\{9\}\} \\
& \mathcal{A}_{1}^{A}=\{\{1\},\{2\},\{3,7\},\{4\},\{5\},\{6\},\{8\},\{9\}\} \\
& \mathcal{A}_{2}^{A}=\{\{1\},\{2\},\{3,7,8\},\{4\},\{5\},\{6\},\{9\}\} \\
& \mathcal{A}_{3}^{A}=\{\{1\},\{2\},\{3,6,7,8\},\{4\},\{5\},\{9\}\} \\
& \mathcal{A}_{4}^{A}=\{\{1,3,6,7,8\},\{2\},\{4\},\{5\},\{9\}\} \\
& \mathcal{A}_{5}^{A}=\{\{1,3,6,7,8,9\},\{2\},\{4\},\{5\}\} \\
& \mathcal{A}_{6}^{A}=\{\{1,2,3,6,7,8,9\},\{4\},\{5\}\} \\
& \mathcal{A}_{7}^{A}=\{\{1,2,3,5,6,7,8,9\},\{4\}\} \\
& \mathcal{A}_{8}^{A}=\{\{1,2,3,4,5,6,7,8,9\}\} .
\end{aligned}
$$

The clusters of candidates associated with the above ordinal proximity measures are illustrated in Figure 4, Figure 5 and Figure 6, which display hierarchical relationships among presidential candidates.

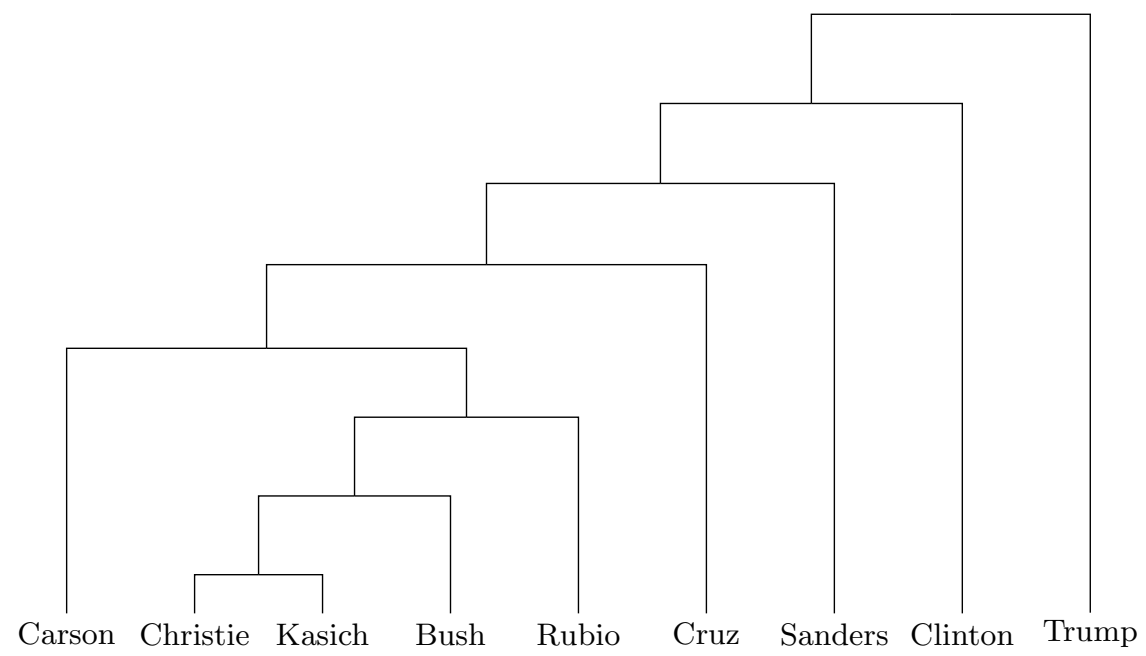

Fig. 4. Clustering tree obtained from the matrix $A_{2222}$ 


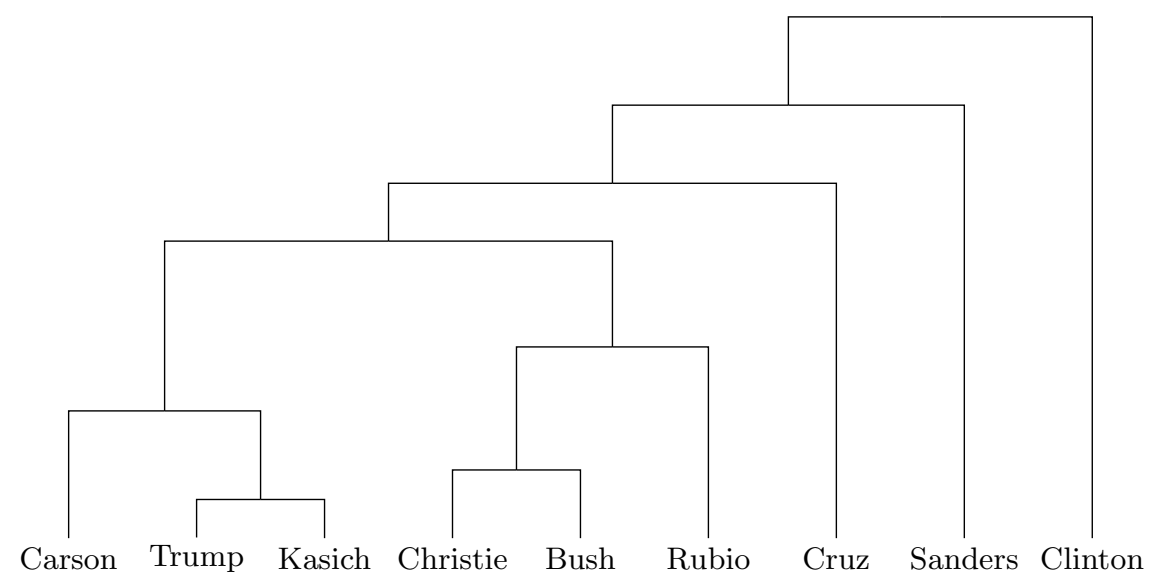

Fig. 5. Clustering tree obtained from the matrix $A_{2332}$

As seen in the above results obtained using the proposed agglomerative hierarchical clustering, the outcomes associated with matrices $A_{2222}$ and $A_{3223}$ are very similar. However, the way that merge the candidates Bush and Rubio is different in each case. Therefore, the results from the three clustering processes developed are distinct depending on the ordinal proximity measure used.

On the other hand, it is interesting to note that the candidate Kasich always appears in the first merged cluster in all considered cases, and the Democratic U.S. presidential candidate Hillary Clinton and her Republican rival Donald Trump are in different clusters throughout the process and they only merge in the final step.

\section{Concluding remarks}

Appraisals of presidential candidates given by voters are considerably influential during presidential campaigns. However, to date, these appraisals have been barely considered in clustering procedures. In this paper, we have developed an agglomerative hierarchical clustering through linguistic appraisal of U.S. 2016 presidential candidates made by a sample of voters.

From the results of the clustering procedure, it should be noted the importance of determining an appropriate ordinal proximity measure, since the way in which elements or clusters are merged may change depending on the ordinal proximity measure used. This issue is addressed in García-Lapresta et al. [6].

As further research, it might to be possible to extend this clustering process based on consensus to multi-criteria decision-making, in such a way that each criterion requires a clustering procedure. 


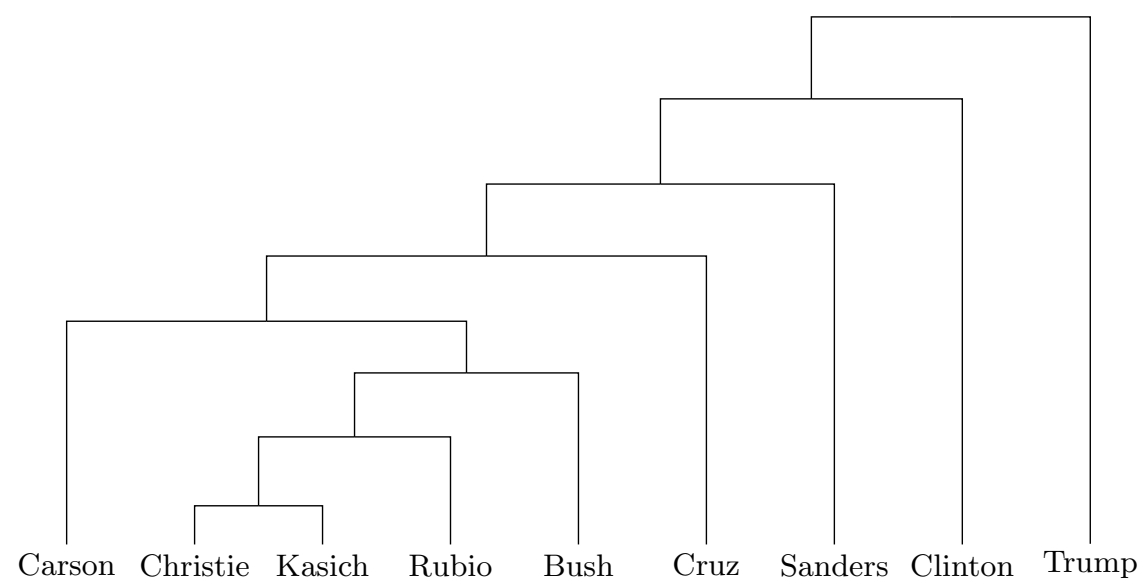

Fig. 6. Clustering tree obtained from the matrix $A_{3223}$

\section{Acknowledgments}

The authors are grateful to Victoriano Ramírez for the information about the data source used in this paper. The financial support from the Spanish $M i$ nisterio de Economía y Competitividad (project ECO2016-77900-P) and ERDF are acknowledged.

\section{References}

1. Aleskerov, F., Nurmi, H.: A method for finding patterns of party support and electoral change: An analysis of British general and Finnish municipal elections. Mathematical and Computer Modelling, pp. 1225-1269 (2008).

2. Balinski, M., Laraki, R.: A theory of measuring, electing and ranking. Proceedings of the National Academy of Sciences of the United States of America 104, pp. 8720-8725 (2007).

3. Balinski, M., Laraki, R.: Majority Judgment. Measuring, Ranking, and Electing. The MIT Press, Cambridge MA (2011).

4. Dowdle, A., Limbocker, S., Yang, S., Sebold, K., Stewart, P.: The Invisible Hands of Political Parties in Presidential Elections: Party Activists and Political Aggregation from 2004 to 2012, Palgrave Macmillan US (2013).

5. Everitt, B.S., Landau, S., Leese, M., Stahl, D.: Cluster Analysis, Wiley (2011).

6. García-Lapresta, J.L., González del Pozo, R., Pérez-Román, D.: Metrizable ordinal proximity measures and their aggregation, submitted.

7. García-Lapresta, J.L., Pérez-Román, D.: Consensus-based hierarchical agglomerative clustering in the context of weak orders. Proceedings of the 2013 IFSA-NAFIPS Joint Congress, Edmonton, pp. 1010-1015 (2013).

8. García-Lapresta, J.L., Pérez-Román, D.: Ordinal proximity measures in the context of unbalanced qualitative scales and some applications to consensus and clustering. Applied Soft Computing 35, pp. 864-872 (2015). 
9. García-Lapresta, J.L., Pérez-Román, D.: Consensus-based clustering under hesitant qualitative assessments. Fuzzy Sets and Systems, pp. 261-273 (2016).

10. García-Lapresta, J.L., Pérez-Román, D.: A consensus reaching process in the context of non-uniform ordered qualitative scales. Fuzzy Optimization and Decision Making (2016). doi: 10.1007/s10700-016-9256-6.

11. Pearson, P.T.,Cooper, C.I.: Using Self Organizing Maps to Analyze Demographics and Swing State Voting in the 2008 U.S. Presidential Election. Vol. 7477 Lecture Notes in Artificial Intelligence, Springer (2012).

12. Pew Research Center. http://www.pewresearch.org

13. Seabrook, N.R.: The Obama effect: Patterns of geographic clustering in the 2004 and 2008 presidential elections. The Forum 7 (2009). doi:10.2202/1540-8884.1308. 